\title{
Low-temperature thermal conductivity of solid carbon dioxide
}

\author{
V.V. Sumarokov, P. Stachowiak, and A. Jeżowski \\ Institute for Low Temperatures and Structure Research of the Polish Academy of Sciences \\ P.O. Box 1410, 50-950 Wrocław, Poland \\ E-mail:p_stach@int.pan.wroc.pl \\ Received May 4, 2003
}

\begin{abstract}
Preliminary results of the thermal conductivity measurements performed in the temperature range $1.5-35 \mathrm{~K}$ on pure carbon dioxide are presented. The data below $25 \mathrm{~K}$ have been obtained for the first time. The thermal conductivity coefficient reaches very high value, of about $700 \mathrm{~W} /(\mathrm{m} \cdot \mathrm{K})$, unusual for simple molecular crystal. Straightforward analysis applied to the data indicates the case of large-grained sample.
\end{abstract}

PACS: 66.70.+f

Solid carbon dioxide belongs to the group of simple molecular crystals formed from linear molecules $\left(\mathrm{N}_{2}\right.$, $\mathrm{CO}, \mathrm{CO}_{2}$ and $\mathrm{N}_{2} \mathrm{O}$ belong to the same group). At equilibrium vapor pressure $\mathrm{CO}_{2}$ crystal has unchanged fcc structure in the whole range of its existence $[1,2]$. In the solid phase the axes of $\mathrm{O}-\mathrm{C}-\mathrm{O}$ molecules, whose carbon atoms are located in the sites of the lattice, are oriented along space diagonals of the cubic elementary cell ( $P a 3$ space group).

Thermal, optical and other properties of solid $\mathrm{CO}_{2}$ have been studied over a wide range of temperature and pressure, see, e.g., [1,2] and references therein. However, the thermal conductivity at equilibrium vapor pressure was investigated only for temperatures above $27 \mathrm{~K}$ [3]. Extension of investigation of the thermal conductivity of solid $\mathrm{CO}_{2}$ towards lower temperatures is of interest by itself. Besides that, a comparison with the results obtained earlier for other simple molecular crystals would provide new information concerning various internal processes in the crystals.

This work presents preliminary results of measurements of the thermal conductivity of solid carbon dioxide in the temperature range $1.5-36 \mathrm{~K}$.

\section{Experimental}

The crystal of $\mathrm{CO}_{2}$ was grown, thermally treated and measured in the home-designed liquid He experimental setup, described fully in [4].
The central part of the setup is a cylindrical glass ampoule which, during the experiment, contains the sample under investigation. The ampoule, of length of $36 \mathrm{~mm}$, has inner diameter of $4.2 \mathrm{~mm}$ and the wall thickness of $1 \mathrm{~mm}$. Two germanium resistance thermometers are attached to the wall of the ampoule by means of thin cooper rings and heat-conducting glue. During measurements the thermometers serve the purpose of temperature and temperature gradient determination. The distance between the thermometers is $12 \mathrm{~mm}$ and the lower thermometer is positioned $\sim 9 \mathrm{~mm}$ from the bottom of the ampoule.

The $\mathrm{CO}_{2}$ crystal was obtained from gas of natural isotopic composition and of $99.999 \%$ chemical purity; the purity of the gas was checked by means of a mass spectrometer. The sample of solid $\mathrm{CO}_{2}$ was grown from gaseous phase, starting at the bottom of the ampoule, with the following conditions of condensation: the temperature $\sim 173.3 \mathrm{~K}$, the pressure $\sim 14 \mathrm{kPa}$, the growth rate $\sim 1.5 \mathrm{~mm} / \mathrm{h}$. During the sample growth the temperature gradient of $\sim 2.2 \mathrm{~K} / \mathrm{cm}$ along the ampoule was maintained. When the crystal fully filled the ampoule, the growth process of the sample was terminated and the temperature gradient was reduced to about $1.1 \mathrm{~K} / \mathrm{cm}$. Next, the sample was cooled. The cooling rate of the sample was about $0.1 \mathrm{~K} / \mathrm{h}$ in the temperature range $173-100 \mathrm{~K}, \sim 0.2 \mathrm{~K} / \mathrm{h}$ in the range $100-70 \mathrm{~K}$ and $\sim 0.5 \mathrm{~K} / \mathrm{h}$ below $70 \mathrm{~K}$. At liquid helium temperatures the sample was transparent without any visible defects or voids. When the sample was at 
the temperature of $\sim 4.2 \mathrm{~K}$, before the measurements start, gaseous helium at pressure of $\sim 1 \mathrm{kPa}$ was admitted to the ampoule to improve thermal contact of the sample with the two thermometers and the gradient heater. Measurements of the thermal conductivity of $\mathrm{CO}_{2}$ were performed by steady state flow method. The experimental error did not exceed $10 \%$.

Other detail of the experiment has been described in Refs. 4-6.

\section{Results and discussion}

The results of the measurements of the thermal conductivity coefficient dependence on temperature for solid $\mathrm{CO}_{2}$ in the temperature range $1.5-36 \mathrm{~K}$ have been depicted in Fig. 1. In the figure earlier high-temperature carbon dioxide data of Koloskova et al. [3] have also been included.

In terms of quality, the dependence of the thermal conductivity on temperature, $\kappa(T)$, obtained in the experiment is typical for a dielectric crystal, see, e.g., $[1,2]$. Initially, in the lowest temperatures, the thermal conductivity increases with increasing temperature, then at temperature around $5 \mathrm{~K}$ the coefficient attains its maximum value. For temperatures corre-

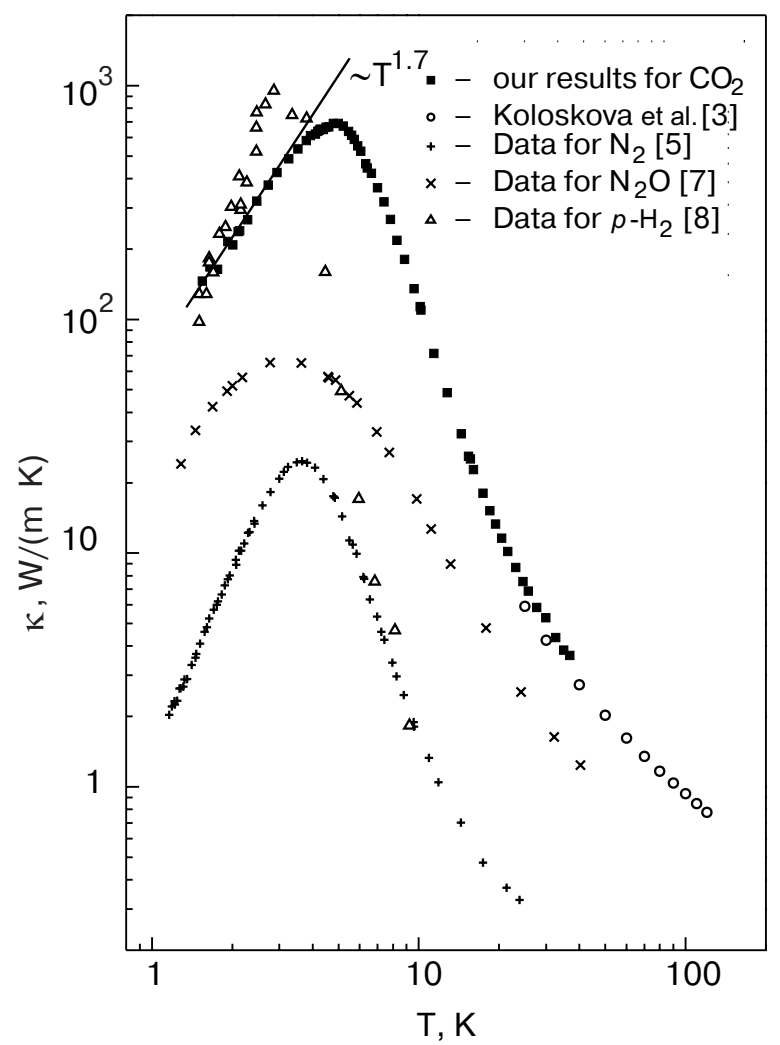

Fig. 1. Temperature dependence of thermal conductivity coefficient of solid carbon dioxide. Earlier data for nitrogen, para-hydrogen and nitrous oxide crystals have also been included. sponding those above the maximum, the thermal conductivity initially quickly decreases, following the exponential decay, and then the decrease becomes weaker. The maximum value of the thermal conductivity coefficient $\kappa$ of $720 \mathrm{~W} /(\mathrm{m} \cdot \mathrm{K})$ should be noticed. This value is much greater than any of those for other (so-far investigated) simple molecular crystals [5-8], except the solid para-hydrogen [7] - for comparison also the data obtained earlier for crystals of pure nitrogen [5], nitrous oxide [7] and para-hydrogen [8] have been depicted in Fig. 1.

Despite high maximum value of the thermal conductivity, which can be regarded as an evidence for good quality of the investigated carbon dioxide crystal and high chemical purity of the sample, the dependence $\kappa(T)$ in low temperatures is far from typical for low-defect-content dielectric crystal. In the standard case of a dielectric crystal with low density of point defects and dislocations, the low-temperature thermal conductivity is specified by scattering of phonons on grain or sample boundaries, which (being independent from the phonon frequency) give $\kappa \sim T^{3}$. In the case of our $\mathrm{CO}_{2}$ sample the dependence is $\kappa \sim T^{1.7}$. This dependence is close to that observed in case of prevailing scattering of phonons on dislocation strain fields [9]. Assuming predominating in low temperatures scattering of phonons on dislocations in $\mathrm{CO}_{2}$ and using the information on crystal structure obtained from analysis of nitrogen thermal conductivity data [5] (where boundary scattering dominated in low temperatures) one can estimate the order of the grain size of the investigated carbon dioxide crystal, as follows. From the thermal conductivity expression for a gas, $\kappa=$ $=1 / 3 C v l$ (where $C$ stands for the specific heat of phonon gas, $v$ for the velocity of propagation of phonons and $l$ for the phonon mean free path - in this particular case limited by the sample grains size) one gets the grain size of the $\mathrm{CO}_{2}$ sample $\sim 10 \mathrm{~mm}$. The above result has been obtained by assuming specific heat of $\mathrm{N}_{2}$ about ten times greater than that of $\mathrm{CO}_{2}$ [1], the same phonon propagation velocity in nitrogen and in carbon dioxide [1], and by taking (from the experiments) the ratio $\kappa_{\mathrm{CO}_{2}} / \kappa_{\mathrm{N}_{2}} \approx 100$, and the nitrogen crystal grain size of the order of $\sim 10^{-2} \mathrm{~mm}$ (from Ref. 5). It means that in our experiment we dealt with a large-grained sample, possibly with a single crystal.

High thermal conductivity in higher temperatures may result from relatively low anharmonicity of $\mathrm{CO}_{2}$ molecule translational vibrations and, therefore, weak phonon-phonon interaction.

More detailed analysis of the results of measurements of the dependence of thermal conductivity on temperature for solid carbon dioxide will be published elsewhere. 
1. Physics of Cryocrystals, Yu.A. Freiman and V.G. Manzhelii (eds.), AIP, New York (1996).

2. V.G. Manzhelii, A.I. Prochvatilov, V.G. Gavrilko, and A.I. Isakina, Structure and Thermodynamic Properties of Cryocrystals, Begell House, New York (1998).

3. L.A. Koloskova, I.N. Krupskii, V.G. Manzhelii, B.Ya. Gorodilov, and Yu.G. Kravchenko, Fiz. Tverd. Tela 16, 3089 (1974) [Sov. Phys. Solid State 16, 1993 (1974)]; Fiz. Kondens. Sost., Institute for Low Temperature Physics and Engeneering (Kharkov), USSR, No. 31 (1974), p. 69.

4. A. Jeżowski and P. Stachowiak, Cryogenics 32, 601 (1992).
5. P. Stachowiak, V.V. Sumarokov, J. Mucha, and A. Jeżowski, Phys. Rev. B50, 543 (1994).

6. P. Stachowiak, V.V. Sumarokov, J. Mucha, and A. Jeżowski, J. Low Temp. Phys. 111, 379 (1998).

7. P. Stachowiak, V.V. Sumarokov, J. Mucha, and A. Jeżowski, The Conference on Cryocrystals and Quatum Crystals, Freising'2002, poster presentation.

8. O.A. Korolyuk, B.Ya. Gorodilov, A.I. Krivchikov, and V.G. Manzhelii, Fiz. Nizk. Temp. 25, 944 (1999) [Low Temp. Phys. 25, 708 (1999)].

9. R. Berman, Thermal Conduction in Solids, Clarendon, Oxford (1976). 